\title{
Dark current and photoresponse characteristics of extended wavelength infrared photodetectors
}

\author{
D. Chauhan, ${ }^{1}$ A. G. U. Perera, ${ }^{1, a)}$ L. H. Li, ${ }^{2}$ L. Chen, ${ }^{2}$ and E. H. Linfield ${ }^{2}$ \\ ${ }^{1}$ Center for Nano-Optics (CeNO), Department of Physics and Astronomy, Georgia State University, Atlanta, \\ Georgia 30303, USA \\ ${ }^{2}$ School of Electronic and Electrical Engineering, University of Leeds, Leeds LS2 9JT, United Kingdom
}

(Received 28 April 2017; accepted 24 June 2017; published online 10 July 2017)

\begin{abstract}
The dark current and spectral photoresponse thresholds of a semiconductor photodetector are normally determined by the minimum energy gap $(\Delta)$ of the material, or the interfacial energy gap of the heterostructure. In this manuscript, we discuss the performance of an asymmetric p-GaAs/ $\mathrm{Al}_{\mathrm{x}} \mathrm{Ga}_{1-\mathrm{x}} \mathrm{As}$ heterostructure-based infrared photodetector, which shows an extended wavelength threshold beyond the limit set by $\Delta$. The measured dark current was found to agree well with fits obtained from a 3D carrier drift model using the designed value of $\Delta \sim 0.40 \mathrm{eV}(\sim 3.1 \mu \mathrm{m})$. In contrast, the spectral photoresponse showed extended wavelength thresholds of $\sim 68 \mu \mathrm{m}, \sim 45 \mu \mathrm{m}$, and $\sim 60 \mu \mathrm{m}$ at positive, zero, and negative biases, respectively, at $5.3 \mathrm{~K}$. For a reference (symmetric) photodetector, the dark current was fitted with the designed value of $\Delta \sim 0.30 \mathrm{eV}$, and excellent agreement was obtained for both the measured dark current and spectral response. This underlies the advantage of using asymmetric infrared photo-detector designs, in which an extension to the detected wavelengths can be obtained with little compromise to the dark current characteristics. Published by AIP Publishing. [http://dx.doi.org/10.1063/1.4992075]
\end{abstract}

\section{INTRODUCTION}

When designing an infrared (IR) photodetector, a fundamental parameter is the minimum energy gap $(\Delta)$ of the IR radiation absorbing material, or the interfacial energy gap of the heterostructure. This gives rise to the conventional wavelength threshold $\left(\lambda_{t}\right)$ of the spectral photoresponse through the relation ${ }^{1} \lambda_{t}=h c / \Delta$. An IR photodetector with the desired spectral photoresponse threshold can thus be designed by choosing a suitable value of $\Delta$. However, lowering the value of $\Delta$ to extend $\lambda_{t}$ comes with the cost of increased dark current and related noise levels. Therefore, development of IR photodetectors, which overcome the restriction imposed on $\lambda_{t}$ by $\Delta$, is of paramount importance since longer wavelength detection is required with little or no compromise to the dark current levels.

In p-type semiconductor materials such as p-type doped GaAs, IR absorption leads to hole transitions from the lighthole/heavy-hole bands to the split-off band, and from the heavy-hole to light-hole bands. Additionally, free carrier absorption, which is weaker at shorter wavelengths, but increases as $\lambda^{2}$ also occurs, ${ }^{2}$ resulting in photo-excitation in the heavy-hole band. Therefore, a p-GaAs layer absorbs a broad IR spectrum spanning short-wave IR (SWIR) to the very-long-wave IR (VLWIR) range. Whilst values of $\Delta$ can be adjusted to cover the mid-IR to far-IR spectral ranges, ${ }^{3,4}$ $\lambda_{t}$ for the spectral photoresponse of $\mathrm{p}-\mathrm{GaAs} / \mathrm{Al}_{\mathrm{x}} \mathrm{Ga}_{1-\mathrm{x}} \mathrm{As}$ heterostructures-based IR photodetectors is still traditionally determined by the interfacial energy gap $(\Delta)$ at the emitter (absorber)/collector barrier interface.

Recently, optoelectronic devices based on hot-carrier driven effects have received broad attention owing to their

\footnotetext{
a) Author to whom correspondence should be addressed: uperera@gsu.edu
}

breadth of potential applications in areas ranging from photodetectors to solar energy harvesting. ${ }^{5-7}$ For example, a hot-carrier based IR photodetector with wavelength tunability, but independent of the semiconductor bandgap, was explored as one candidate for IR photodetectors. ${ }^{8}$ In another study, ${ }^{9}$ VLWIR detection (up to $\sim 55 \mu \mathrm{m}$ ) was demonstrated with a photodetector in which the designed value of $\Delta$ was close to $\sim 3.9 \mu \mathrm{m}$. In this case, asymmetrical p-GaAs $/ \mathrm{Al}_{\mathrm{x}} \mathrm{Ga}_{1-\mathrm{x}} \mathrm{As}$ heterostructures were used, where the $\mathrm{p}-\mathrm{GaAs}$ layer, sandwiched by $\mathrm{Al}_{\mathrm{x}} \mathrm{Ga}_{1-\mathrm{x}} \mathrm{As}$ barrier layers, works both as an absorber and emitter.

The underlying mechanism leading to this VLWIR photoresponse is based on hot carrier effects. Photo-excited carriers have an energy greater than those in thermal equilibrium, ${ }^{7}$ and undergo relaxation processes dominated by optical phonon emission. ${ }^{9,10}$ However, a phonon-bottleneck $^{5,11,12}$ alters the hot carrier relaxation path towards an efficient energy transfer to cold carriers. ${ }^{5}$ In $\mathrm{p}-\mathrm{GaAs} / \mathrm{Al}_{\mathrm{x}} \mathrm{Ga}_{1-}$ ${ }_{x}$ As heterostructures, high energy carriers (holes) can be created by photo-injection over a high energy barrier into the emitter, which causes a transient energy redistribution, leading to elevation of the Fermi level. The energy difference between the Fermi level in the emitter and the height of the emitter-collector barrier thus becomes smaller, reducing the activation energy for holes to pass to the collector. As a result, a photoresponse can be observed over an extended wavelength range, ${ }^{9}$ that is, beyond the limit set by $\Delta$. However, to achieve such an extended wavelength photoresponse, an energy offset $(\delta E)$ between the injector-emitter and emitter-collector barriers is necessary. ${ }^{13}$ At high values of $\delta E$, the detector then operates under both positive and negative applied biases, and also in a photovoltaic mode. ${ }^{13}$

In this paper, we present a study of the dark current of an extended wavelength photodetector, in the temperature range 
$10-100 \mathrm{~K}$. We find that the fit of the measured dark current, obtained through a 3D carrier drift model, agrees well with the designed value of $\Delta \sim 0.40 \mathrm{eV}(\sim 3.1 \mu \mathrm{m})$. In contrast, a photoresponse was observed up to $\sim 45 \mu \mathrm{m}$ at $5.3 \mathrm{~K}$ at zero bias, and up to $\sim 60 \mu \mathrm{m}$ and $\sim 68 \mu \mathrm{m}$ in the negative and positive bias regimes, respectively. A reference detector without energy offset $(\delta E)$ does not show an extended wavelength threshold, but a good agreement with the dark current fits is observed with the designed value of $\Delta \sim 0.30 \mathrm{eV}$.

\section{DEVICE DESIGN AND CHARACTERIZATION}

Two p-GaAs/ $\mathrm{Al}_{\mathrm{x}} \mathrm{Ga}_{1-\mathrm{x}} \mathrm{As}$ heterostructures (15SP3 and LH1002) were grown by molecular beam epitaxy on a semiinsulating GaAs substrate. The valence band alignment of these heterostructures is shown schematically in Fig. 1 at zero bias. The active region of the photodetector consists of an $\mathrm{Al}_{\mathrm{x}} \mathrm{Ga}_{1-\mathrm{x}} \mathrm{As}$ barrier, followed by a $\mathrm{p}$-GaAs emitter, and then another $\mathrm{Al}_{\mathrm{x}} \mathrm{Ga}_{1-\mathrm{x}} \mathrm{As}$ barrier. These emitter/barrier layers are sandwiched by the bottom and top contact layers of ptype doped GaAs, with thicknesses of $0.5 \mu \mathrm{m}$ and $0.2 \mu \mathrm{m}$, respectively. The emitters, and the top and bottom contact layers, are degenerately doped at $1 \times 10^{19} \mathrm{~cm}^{-3}$. The thicknesses of the emitters in both structures are sufficiently large to have a bulk-like distribution of energy states.

Sample LH1002 consists of a $20 \mathrm{~nm}$ emitter and two $60 \mathrm{~nm} \mathrm{Al} l_{0.57} \mathrm{Ga}_{0.43} \mathrm{As}$ barriers. Since there is no energy offset $(\delta E)$ between the two barriers, LH1002 is used as the reference detector in this study. Sample 15SP3 consists of an $80 \mathrm{~nm}$ emitter, a constant $\mathrm{Al}_{0.39} \mathrm{Ga}_{0.61} \mathrm{As}$ barrier $(400 \mathrm{~nm})$ at the top, whilst the $\mathrm{Al}$ mole fraction in the bottom $\mathrm{Al}_{\mathrm{x}} \mathrm{Ga}_{1-\mathrm{x}} \mathrm{As}$ barrier is linearly tuned from $\mathrm{x}_{1}=0.45$ at the bottom to $\mathrm{x}_{2}=0.75$ at the top to create a graded barrier. The values of the Al mole fractions chosen in the two barriers lead to an energy offset $(\delta E)$ of $\sim 0.19 \mathrm{eV}$ between the graded and the constant barrier. The details of the sample parameters and spectral photoresponse thresholds are summarized in Table I. Mesas with an electrically active area of $400 \times 400 \mu \mathrm{m}^{2}$ were fabricated by conventional photolithography and wet etching, and the contact electrodes were formed by Ti/Pt/Au metallization. By partially etching the top contact layer (p-GaAs), an optical window of $\sim 260 \times 260 \mu \mathrm{m}^{2}$ was opened for normal incidence optical illumination of the detector.

The current-voltage characteristics of the photodetectors were measured using a Keithley 2400 source meter. A positive voltage connected to the top contact with the bottom contact grounded is referred to as a positive bias. Similarly, a positive voltage connected to the bottom contact with the top contact grounded is referred to as a negative bias. A Fourier transform infrared (FTIR) spectrometer was used to measure the spectral photoresponse, and a commercial $\mathrm{Si}$ composite bolometer with known sensitivity was used to measure the background intensity of a blackbody source to calibrate the photoresponse.

\section{RESULTS AND DISCUSSION}

The measured dark current of the photodetector LH1002 is shown in Fig. 2(a) (dashed lines) and the solid lines represent the fits, in the temperature range of $10-100 \mathrm{~K}$, based on a $3 \mathrm{D}$ carrier drift model, ${ }^{14}$ given by the relation

$$
I_{\text {dark }}=\operatorname{Aep}_{3 D} v(F) \text {. }
$$

Here, $A$ is the electrically active area of the detector, $e$ is the electronic charge, $p_{3 D}$ is the thermally-excited carrier (hole) density on the top of the barrier, and $v(F)$ is the hole drift velocity as a function of applied field $F$. Using $p_{3 D}$ and $v(F)$ given by ${ }^{14}$

$$
p_{3 D}=2\left(\frac{m^{*} k_{B} T}{2 \pi \hbar^{2}}\right)^{3 / 2} \exp \left(-\frac{E_{a c t}}{k_{B} T}\right)
$$

and

$$
v(F)=\frac{\mu F}{\left(1+\left(\frac{\mu F}{v_{\text {sat }}}\right)^{2}\right)^{1 / 2}} .
$$

Equation (1) reduces to the familiar form ${ }^{14,15}$

$$
\begin{aligned}
I_{\text {dark }}= & A e \frac{\mu F}{\left[1+\left(\frac{\mu F}{v_{\text {sat }}}\right)^{2}\right]^{1 / 2}} 2\left(\frac{m^{*} k_{B} T}{2 \pi \hbar^{2}}\right)^{3 / 2} \\
& \times \exp \left(-\frac{\Delta-\alpha F-E_{f}}{k_{B} T}\right) .
\end{aligned}
$$
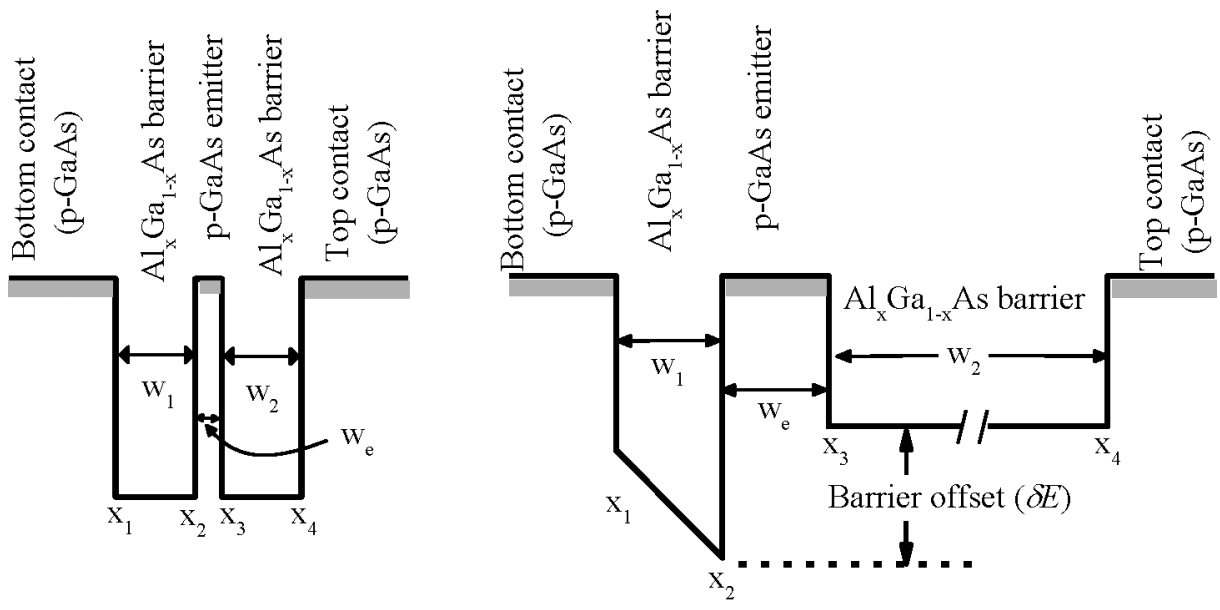

(a) (b)
FIG. 1. Schematic diagrams of the valence band alignment of the detectors in equilibrium: (a) LH1002 $(\Delta \sim$ $0.30 \mathrm{eV})$ consists of an emitter $(20 \mathrm{~nm})$ and two $\mathrm{Al}_{0.57} \mathrm{Ga}_{0.43} \mathrm{As}$ barriers $(60 \mathrm{~nm})$, with no energy offset between the barriers. (b) $15 \mathrm{SP} 3(\Delta \sim 0.40 \mathrm{eV})$ consists of an emitter $(80 \mathrm{~nm})$, an $\mathrm{Al}_{\mathrm{x}} \mathrm{Ga}_{1-\mathrm{x}}$ As barrier $(80 \mathrm{~nm})$ at the bottom graded by tuning the $\mathrm{Al}$ mole fraction from $\mathrm{x}_{1}=0.45$ to $\mathrm{x}_{2}=0.75$, and a $400 \mathrm{~nm} \mathrm{Al}_{0.39} \mathrm{Ga}_{0.61} \mathrm{As}$ barrier at the top; there is thus an energy offset $(\delta E)$ of $\sim 0.19 \mathrm{eV}$ between the two barriers. The emitters are sufficiently thick to have a bulk-like distribution of energy states. For all samples, the emitter, top, and bottom contacts are p-type doped at $1 \times 10^{19} \mathrm{~cm}^{-3}$. 
TABLE I. Summary of sample parameters and wavelength thresholds. All emitters, and the top and bottom contacts (p-GaAs), are doped to $1 \times 10^{19} \mathrm{~cm}^{-3}$.

\begin{tabular}{lccccccc}
\hline \hline Sample & $\begin{array}{c}\Delta(\mathrm{eV}) \\
(\text { designed })\end{array}$ & $\begin{array}{c}\text { Energy offset } \\
(\delta E)(\mathrm{eV})\end{array}$ & $\begin{array}{c}\text { Al mole fraction } \\
\mathrm{x}_{1}, \mathrm{x}_{2}, \mathrm{x}_{3}=\mathrm{x}_{4}\end{array}$ & $\mathrm{w}_{\mathrm{e}}(\mathrm{nm})$ & $\mathrm{w}_{1}(\mathrm{~nm})$ & $\mathrm{w}_{2}(\mathrm{~nm})$ & $\begin{array}{c}\text { Wavelength } \\
\text { threshold }\left(\lambda_{\mathrm{t}}\right) \text { at } 5.3 \mathrm{~K}\end{array}$ \\
\hline 15SP3 & 0.40 & 0.19 & $0.45,0.75,0.39$ & 80 & 80 & 400 & $60 \mu \mathrm{m},-1 \mathrm{~V} ; \sim 68 \mu \mathrm{m}, 4 \mathrm{~V} ; \sim 45 \mu \mathrm{m}, 0 \mathrm{~V}$ \\
LH1002 & 0.30 & None & $0.57,0.57,0.57$ & 20 & 60 & 60 & $\sim 4 \mu \mathrm{m},-0.2 \mathrm{~V}(\mathrm{Ref} .13)$ \\
\hline \hline
\end{tabular}

Here, $E_{a c t}=\Delta-\alpha F-E_{f}$ is the activation energy, $\mu$ is the mobility of the holes, $v_{\text {sat }}$ is the saturation velocity, $m^{*}$ is the effective mass, $k_{B}$ is Boltzmann's constant, $T$ is the temperature, $\hbar$ is the reduced Planck constant, $\alpha$ is a fitting parameter that determines effective barrier lowering due to the applied field, and $E_{f}$ is the Fermi level. In the p-GaAs layers, the mobility of the holes $\left(\mu_{p-G a A s}\right)$ will be much smaller due to high carrier-impurity scattering, compared to that in undoped $\mathrm{Al}_{\mathrm{x}} \mathrm{Ga}_{1-\mathrm{x}} \mathrm{As}$ layers $\left(\mu_{\text {AlGaAs }}\right)$. Thus, the effective mobility is equivalent to $\mu_{p-G a A s}$ within an approximation of the Matthiessen rule: ${ }^{1} \mu \approx\left(1 / \mu_{p-\text { GaAs }}+1 / \mu_{\text {AlGaAs }}\right)^{-1}$. For degenerately doped p-GaAs, $E_{f}$ is given by ${ }^{1}$
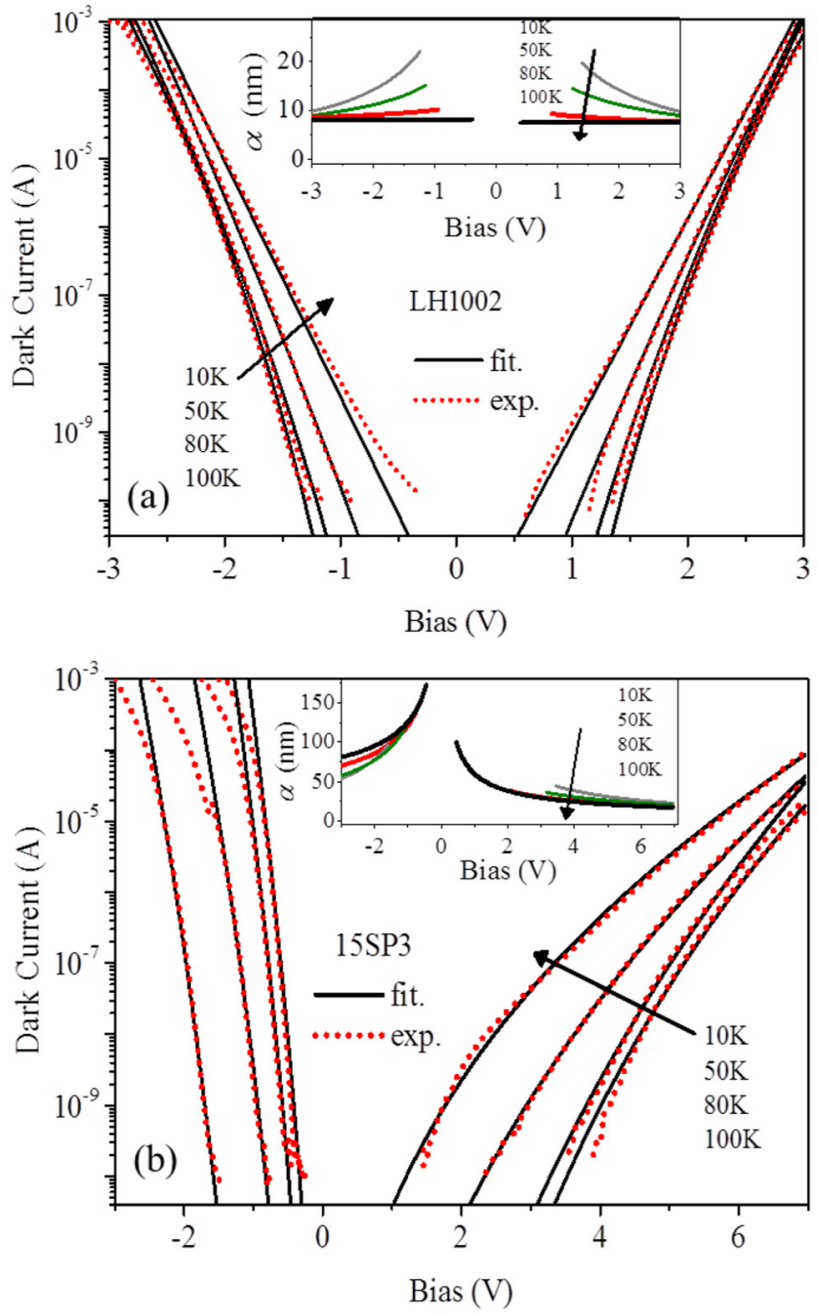

FIG. 2. Experimentally measured and fitted dark current curves in the temperature range $10-100 \mathrm{~K}$ for (a) LH1002 (inset showing the fitting parameter $\alpha$ ), and (b) 15SP3 (inset showing the fitting parameter $\alpha$ ). The dark current curves and the fitting parameters are symmetrical for LH1002, whilst they are non-symmetrical for $15 \mathrm{SP} 3$.

$$
E_{V}-E_{f}=k_{B} T\left[\ln \left(\frac{p}{N_{V}}\right)+2^{-3 / 2}\left(\frac{p}{N_{V}}\right)\right],
$$

where $E_{V}$ is the energy at the valence band-edge, $p$ is the doping density, and $N_{V}$ is the density of states in the valence band given by ${ }^{1}$

$$
N_{V}=2\left(\frac{2 \pi m_{d h} k_{B} T}{h^{2}}\right)^{3 / 2}
$$

By substituting $k_{B}, m_{d h}$ (the density-of-state effective mass for holes), and $h$ (Planck constant), Eq. (6) reduces (for $\left.\mathrm{Al}_{\mathrm{x}} \mathrm{Ga}_{1-\mathrm{x}} \mathrm{As}, \mathrm{x}<0.4\right)$ to ${ }^{16}$

$$
N_{V}=4.82 \times 10^{15}(0.51+0.25 x)^{3 / 2}\left(\mathrm{~cm}^{-3}\right) .
$$

Since both structures (LH1002 and 15SP3) consist of pGaAs doped to the same level, the same values of $\mu$ and $v_{\text {sat }}$ were used at a given temperature. At a doping density of $1 \times 10^{19} \mathrm{~cm}^{-3}, \mu \sim 50 \mathrm{~cm}^{2} / \mathrm{V} \mathrm{s}$ at room temperature, and an empirical temperature dependence is given ${ }^{17}$ as

$$
\mu(T)=\left[2.5 \times 10^{-3}\left(\frac{T}{300}\right)^{2.3}+4 \times 10^{-21} p\left(\frac{300}{T}\right)^{1.5}\right]^{-1}\left(\mathrm{~cm}^{2} / \mathrm{V} \mathrm{s}\right) .
$$

Similarly, $v_{\text {sat }} \sim 10^{7} \mathrm{~cm} / \mathrm{s}$ at room temperature, and an empirical temperature dependence is given ${ }^{18}$ as

$$
v_{\text {sat }}(T)=\frac{v_{\text {sat }, 300 K}}{(1-A)+A \frac{T}{300}}(\mathrm{~cm} / \mathrm{s})
$$

with $A=0.59$ for p-GaAs. $^{18}$ Finally, the parameter $\alpha$ is obtained from fitting of the dark current curves at each temperature.

The fitted dark current curves [solid lines, Fig. 2(a)] agreed reasonably closely with the experimentally measured dark current curves [dashed lines, Fig. 2(a)] in the 10-100 K temperature range for LH1002 with $\Delta \sim 0.30 \mathrm{eV}$. Based on these fits, the value of the parameter $(\alpha)$ for LH1002 was $\sim 10 \mathrm{~nm}$, and had a symmetrical bias-dependence. This value was found to increase at low temperatures and low biases [inset, Fig. 2(a)]. A good agreement was also found between the fitted and measured dark current curves for 15SP3 in the 10-100 K temperature range [Fig. 2(b)]. As seen in the inset of Fig. 2(b), $\alpha$ for 15SP3 has a non-symmetrical bias-dependence, which is expected due to the non-symmetrical design of the heterostructure, and also due to the non-symmetrical bias-dependence of the measured dark current. Deviation of the value of $\alpha$ in the low temperature and low bias range is 
similar to LH1002. Due to the temperature-varying nature of $E_{f}$, and also due to an asymmetrical design of 15SP3, the value of $\alpha$ was allowed to change to obtain reasonable fittings. However, it is important to note that $\Delta \sim 0.40 \mathrm{eV}$, which is the designed value of $\Delta$ for 15SP3, fits the experimental results most closely.

For LH1002, the spectral range of the photoresponse was also in good agreement ${ }^{13}$ with the designed value of $\Delta \sim 0.30 \mathrm{eV}$, due to the absence of an energy offset $(\delta E)$. However, 15SP3 (with an energy offset of $\delta E \sim 0.19 \mathrm{eV}$ ) shows an extended wavelength photoresponse in the VLWIR range: up to $\sim 45 \mu \mathrm{m}$ at zero bias, and up to $\sim 68 \mu \mathrm{m}$ and $\sim 60 \mu \mathrm{m}$ in the positive and negative bias regimes, respectively, at $5.3 \mathrm{~K}$. Typical data is shown in Fig. 3(a) for negative biases of $-0.6,-0.8$, and $-1.0 \mathrm{~V}$ (with the photoresponse at zero bias shown in the inset), and in Fig. 3(b) for positive biases 2, 3, and $4 \mathrm{~V}$. The spectral thresholds are largely unchanged under both negative and positive biases. However, the photoresponse became stronger at increased applied biases, due to an improved collection efficiency. Under a negative bias, hot carriers are injected from the bottom contact to the emitter leading to an energy transfer to the cold carriers that enables the photoresponse in the extended wavelength region. Under a positive bias, hot carriers are injected into the
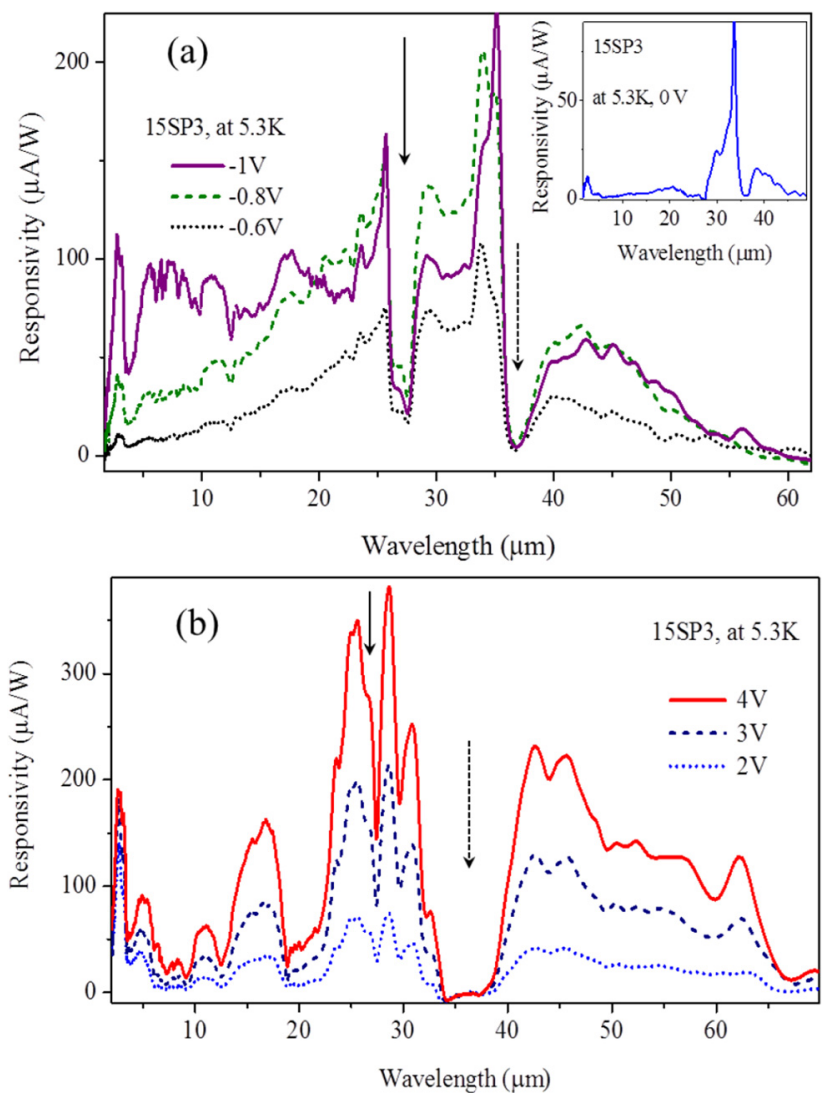

FIG. 3. Spectral photoresponse of the detector $15 \mathrm{SP} 3$ at $5.3 \mathrm{~K}$ : (a) up to $\sim 60 \mu \mathrm{m}$ under a negative bias, (b) up to $\sim 68 \mu \mathrm{m}$ under a positive bias, and up to $\sim 45 \mu \mathrm{m}$ at zero bias [inset, (a)]. For comparison, the designed value of $\Delta$ should give a threshold wavelength of only $\sim 3.1 \mu \mathrm{m}$. The solid and dashed arrows mark features associated with the AlAs-like and GaAs-like phonons, respectively. In comparison to the negative bias data, the AlAslike phonon feature is narrowed under a positive bias, whilst the GaAs-like phonon feature is widened.

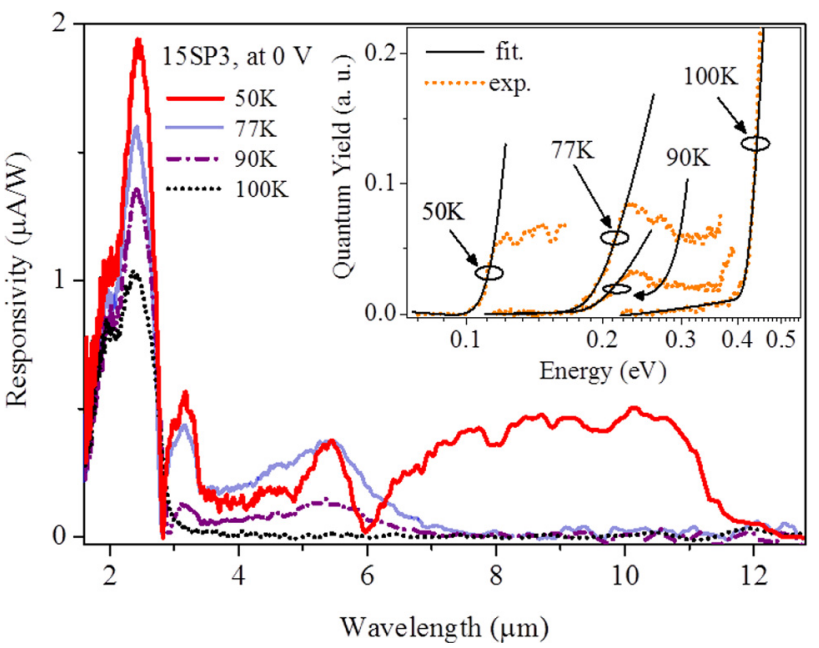

FIG. 4. Spectral photoresponse of the detector $15 \mathrm{SP} 3$ at $0 \mathrm{~V}$, in the temperature range $50-100 \mathrm{~K}$. The extended wavelength threshold was observed to be $\sim 11.5 \mu \mathrm{m}$ at $50 \mathrm{~K}$, and $\sim 7 \mu \mathrm{m}$ at $77 \mathrm{~K}$ and $90 \mathrm{~K}$. The photoresponse in the extended wavelength region disappeared at $100 \mathrm{~K}$, and the threshold of $\sim 3 \mu \mathrm{m}$ then closely agrees with the designed value of $\Delta \sim 0.40 \mathrm{eV}$. Inset: temperature-dependent internal photoemission spectroscopy [TDIPS (Ref 19)] fittings of the spectra to determine the wavelength threshold.

emitter from the top contact. The escape of photo-excited carriers from the emitter towards the bottom contact is less likely due to the high energy of the graded barrier. However, with increasing applied positive biases the graded barrier assumes a sharp triangular shape, and barrier lowering also occurs due to image force effects. ${ }^{19}$ Therefore, carriers escape and collection can be enhanced, with further increases arising from tunneling. This leads to a strong photoresponse in the extended wavelength range under a positive bias as well. It may also be suggested that, by replacing the constant emittercollector barrier by a graded barrier, the photoresponse may improve in the negative bias as well. Additionally, relatively

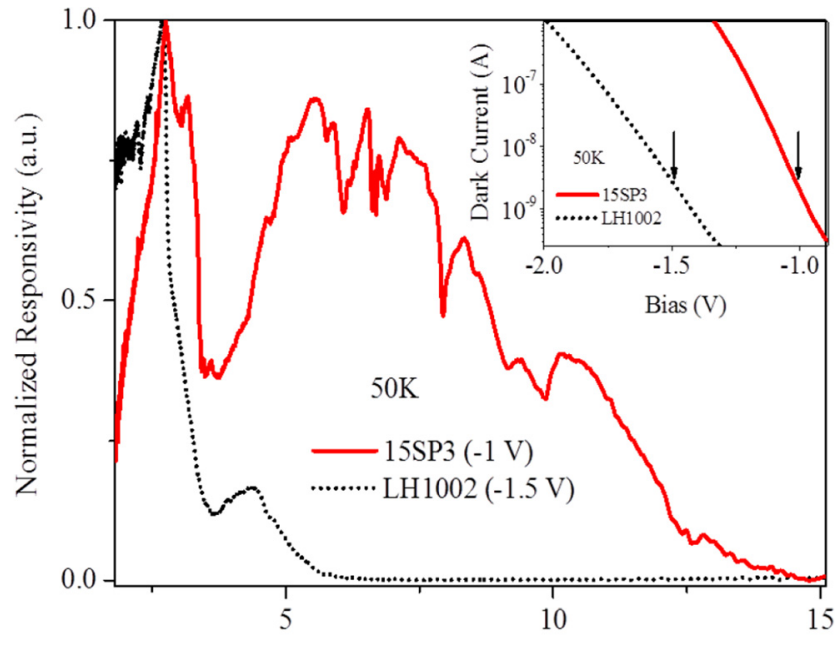

Wavelength $(\mu \mathrm{m})$

FIG. 5. Photoresponse of $15 \mathrm{SP} 2$ and LH1002 as a function of wavelength. At $50 \mathrm{~K}$, the extended wavelength threshold of $15 \mathrm{SP} 3$ is observed up to $\sim 13.5 \mu \mathrm{m}$ at $-1 \mathrm{~V}$, compared to a threshold of $5.5 \mu \mathrm{m}$ of LH1002 at $-1.5 \mathrm{~V}$, whilst the dark current values (inset) are close to each other $\left(\sim 2 \times 10^{-9} \mathrm{~A}\right)$ at those bias values (marked by arrows). 
weak responsivity observed here is believed to be due to a short hot carrier lifetime, typically $\sim 1 \mathrm{ps}^{20}$ Replacing the bulk p-GaAs emitter by nanostructures such as quantum wells and quantum dots is expected to improve the lifetime ${ }^{21}$ and hence the performance.

The sharp peaks observed near $25.5 \mu \mathrm{m}$ and $35.6 \mu \mathrm{m}$ in the photoresponse under a negative bias (marked by solid and dashed arrows) are consistent with previously observed results, and are related to strong plasmon-phonon coupling modes. ${ }^{9}$ Under a positive bias, the AlA-like phonon feature becomes narrower, whilst the GaAs-like phonon feature widens. Even though this difference is not fully understood, it is not unexpected. The hole concentration is $\sim 1 \times 10^{19} \mathrm{~cm}^{-3}$ in equilibrium. Under bias, however, there is a transient concentration of holes, which depends upon the energy of the hot holes injected into the emitter and the hot-cold hole energy transfer ratethese are expected to be significantly different under positive and negative biases, and hence the plasmon-phonon coupling is expected to be different. Further study under non-equilibrium conditions will be necessary to understand more fully the effect of plasmon-phonon coupling on the photoresponse spectra under different operating conditions.

The extension of the photoresponse beyond $\lambda_{t}$ is affected by the operating temperature, ${ }^{13}$ possibly due to a less efficient hot-cold carrier energy transfer mechanism, and a reduced lifetime of the hot carriers with increasing temperature. Figure 4 shows the photoresponse spectra of 15SP3 at zero bias between $50-100 \mathrm{~K}$, with temperature-dependent internal photoemission spectroscopy (TDIPS) ${ }^{19}$ being used to determine the value of the energy $(\Delta)$ corresponding to the spectral threshold of the photoresponse. In this method, $\Delta$ is obtained from fitting the quantum yield, $\left(Y(h \nu) \sim(h \nu-\Delta)^{2}\right)$, in the near-threshold region, as illustrated in the inset of Fig. 4. The wavelength threshold of the photoresponse spectrum is observed to be $\sim 11.5 \mu \mathrm{m}$ at $50 \mathrm{~K}$, and $\sim 7 \mu \mathrm{m}$ at $77 \mathrm{~K}$ and $90 \mathrm{~K}$. At $100 \mathrm{~K}$, however, the photoresponse in the extended wavelength region completely disappears, with the photoresponse being observed only up to $\sim 3 \mu \mathrm{m}$, closely agreeing with the designed value of $\Delta \sim 0.40 \mathrm{eV}$.

The reduced dark current, when using photo-detection in the extended wavelength regime, is further illustrated by the results presented in Fig. 5. The photoresponses of LH1002 and 15SP3 at $50 \mathrm{~K}$ are shown at biases of $-1.5 \mathrm{~V}$ and $-1 \mathrm{~V}$, respectively. At these biases, the dark currents of the two detectors are close to each other $\left(\sim 2 \times 10^{-9} \mathrm{~A}\right.$, as indicated by the arrows in the inset of Fig. 5). However, the photoresponse of 15SP3, with the wavelength threshold extension, is observed up to $\sim 13.5 \mu \mathrm{m}$, as opposed to $5.5 \mu \mathrm{m}$ for LH1002. It should be noted that there is a small increase in the wavelength threshold of LH1002, compared to its designed value, due to image force effects. ${ }^{19}$ The importance of the wavelength threshold extension mechanism is thus clearly illustrated, with its application for future IR photodetector design and development.

\section{CONCLUSION}

We have studied the dark current and photoresponse characteristics of an asymmetric p-GaAs/ $\mathrm{Al}_{\mathrm{x}} \mathrm{Ga}_{1-\mathrm{x}} \mathrm{As}$ heterostructure-based IR photodetector, which shows an extended wavelength threshold beyond the limit of the designed value of $\Delta$. Based on a $3 \mathrm{D}$ carrier drift model, good fits were obtained for the measured dark current in the temperature range of $10-100 \mathrm{~K}$, with $\Delta \sim 0.40 \mathrm{eV}$ $(\sim 3.1 \mu \mathrm{m})$ being used for the fitting. A photoresponse was, however, observed with a wavelength threshold extension up to $\sim 68 \mu \mathrm{m}$ and $\sim 60 \mu \mathrm{m}$ under positive and negative biases, respectively, and up to $\sim 45 \mu \mathrm{m}$ at zero bias, at 5.3 K. An extended wavelength threshold was observed up to $90 \mathrm{~K}$. At $100 \mathrm{~K}$, however, the wavelength threshold of $\sim 3 \mu \mathrm{m}$ agreed closely with the designed value of $\Delta$. The dark current was also measured for a (reference) symmetric $\mathrm{p}-\mathrm{GaAs} / \mathrm{Al}_{\mathrm{x}} \mathrm{Ga}_{1-\mathrm{x}} \mathrm{As}$ heterostructure-based IR photodetector, and no extended wavelength photoresponse was observed. Measured dark currents in the temperature range of $10-100 \mathrm{~K}$ were, however, fit well with $\Delta \sim 0.30 \mathrm{eV}$, close to the design value.

\section{ACKNOWLEDGMENTS}

This work was supported in part by the U.S. Army Research Office under Grant No. W911 NF-15-1-0018, and in part by the National Science Foundation (NSF) under Grant No. ECCS-1232184. Funding was also received from the European Community's Seventh Framework Programme (FP7-IDEAS-ERC) under Grant agreement No. 247375 'TOSCA'. Dilip Chauhan and Edmund Linfield acknowledge support from the GSU Brains and Behavior Fellowship, and the Royal Society and Wolfson Foundation, respectively.

${ }^{1}$ S. M. Sze and K. K. Ng, Physics of Semiconductor Devices, 3rd ed. (John Wiley and Sons, New Jersey, 2007), p. 664, 28, and 19.

${ }^{2}$ A. L. Korotkov, A. G. U. Perera, W. Z. Shen, J. Herfort, K. H. Ploog, W. J. Schaff, and H. C. Liu, J. Appl. Phys. 89(6), 3295 (2001).

${ }^{3}$ S. G. Matsik, M. B. M. Rinzan, A. G. U. Perera, H. C. Liu, Z. R. Wasilewski, and M. Buchanan, Appl. Phys. Lett. 82(1), 139 (2003).

${ }^{4}$ M. B. M. Rinzan, A. G. U. Perera, S. G. Matsik, H. C. Liu, M. Buchanan, G. von Winckel, A. Stintz, and S. Krishna, Infrared Phys. Technol. 47(1-2), 188 (2005).

${ }^{5}$ N. M. Gabor, J. C. W. Song, Q. Ma, N. L. Nair, T. Taychatanapat, K. Watanabe, T. Taniguchi, L. S. Levitov, and P. J. Herrero, Science 334(6056), 648 (2011).

${ }^{6}$ M. Freitag, T. Low, F. Xia, and P. Avouris, Nat. Photonics 7(1), 53-59 (2013).

${ }^{7}$ M. L. Brongersma, N. J. Halas, and P. Nordlander, Nat. Nanotechnol. 10(1), 25 (2015).

${ }^{8}$ Y. Zhan, K. Wu, C. Zhang, S. Wu, and X. Li, Opt. Lett. 40(18), 4261 (2015).

${ }^{9}$ Y. F. Lao, A. G. U. Perera, L. H. Li, S. P. Khanna, E. H. Linfield, and H. C. Liu, Nat. Photonics 8(5), 412 (2014).

${ }^{10}$ D. Sicault, R. Teissier, F. Pardo, J. L. Pelouard, and F. Mollot, Phys. Rev. B 65(12), 121301 (2002).

${ }^{11}$ V. B. Campos and S. D. Sarma, Phys. Rev. B 45(7), 3898 (1992).

${ }^{12}$ V. B. Campos, S. D. Sarma, and M. A. Stroscio, Phys. Rev. B 46(7), 3849 (1992).

${ }^{13}$ D. Chauhan, A. G. U. Perera, L. H. Li, L. Chen, S. P. Khanna, and E. H. Linfield, Opt. Eng. 56(9), 091605 (2017).

${ }^{14}$ E. R. Weber, R. K. Willardson, H. C. Liu, and F. Capasso, Intersubband Transitions in Quantum Wells: Physics and Device Applications (Elsevier Science, 1999), p. 139.

${ }^{15}$ D. G. Esaev, M. B. M. Rinzan, S. G. Matsik, and A. G. U. Perera, J. Appl. Phys. 96(8), 4588 (2004). 
${ }^{16}$ M. Levinshtein, S. Rumyantsev, and M. Shur, Handbook Series on Semiconductor Parameters: Ternary and Quaternary A3B5 Semiconductors (World Scientific, 1999), p. 7.

${ }^{17}$ J. J. S. Blakemore, J. Appl. Phys. 53(10), R123 (1982).

${ }^{18}$ R. Quay, C. Moglestue, V. Palankovski, and S. Selberherr, Mater. Sci. Semicond. Process. 3(1-2), 149 (2000).
${ }^{19}$ Y. F. Lao and A. G. U. Perera, Phys. Rev. B 86(19), 195315 (2012).

${ }^{20}$ S. Gupta, S. Sethi, and P. K. Bhattacharya, Appl. Phys. Lett. 62(10), 1128 (1993).

${ }^{21}$ E. A. Zibik, T. Grange, B. A. Carpenter, N. E. Porter, R. Ferreira, G. Bastard, D. Stehr, S. Winnerl, M. Helm, H. Y. Liu, M. S. Skolnick, and L. R. Wilson, Nat. Mater. 8(10), 803 (2009). 